\title{
Endarterectomia carotídea sob bloqueio loco-regional
}

\author{
Carotid endarterectomy under locoregional anesthesia
}

\section{Ana Terezinha G uillaumon ${ }^{1}$, N ara G elle 0 liveira ${ }^{2}$, Lucas M arcelo D ias Freire ${ }^{2}$, Luis Baldini N eto' ${ }^{2}$, Aline M eira M artins'2, Eduardo Faccini Rocha ${ }^{3}$}

\section{Resumo}

O bjetivo: 0 s autores objetivam descrever os resultados nas cirurgias de endarterectomia carotídea, utilizando-se anestesia loco-regional, emprego seletivo de shunt e proteção farmacológica intra-operatória.

M étodo: Foram estudados doentes submetidos à endarterectomia carotídea sob bloqueio loco-regional, no H ospital das Clínicas da Universidade Estadual de Campinas (UN ICAM P), no período de março de 1996 a maio de 2004. Este estudo é composto de 119 endarterectomias (108 doentes), sendo 69 doentes $(63,8 \%)$ do sexo masculino e $39(36,2 \%)$ do sexo feminino. A idade média foi de 63 anos, variando entre 43 e 83 anos. A indicação da operação foi estenose sintomática em 60 doentes (55,5\%) e assintomática em 48 (44,5\%). Foi realizada avaliação, por imagem, pelo eco-D oppler colorido e arteriografia.

Resultados: Foram excluídos do trabal ho cinco doentes nos quais foi necessária a conversão para a anestesia geral por intolerância à colocação do clampe carotídeo. Dos 103 doentes submetidos a 114 endarterectomias, utilizamos um shunt em quatro cirurgias (3,5\%), em função da intolerância do paciente à colocação do clampe carotídeo durante 0 ato cirúrgico. U tilizou-se remendo em 19 (16,6\%) cirurgias. A mortalidade no período pós-operatório imediato ocorreu em três doentes $(2,6 \%)$, todos em decorrência de acidente vascular cerebral. U m doente $(0,87 \%)$ apresentou acidente vascular isquêmico menor, e um $(0,87 \%)$ doente apresentou síndrome de hiperperfusão, que evoluiu com acidente vascular cerebral hemorrágico. Esse doente foi submetido à drenagem do hematoma cerebral, com recuperação total do déficit neurológico. N o pós-operatório tardio, houve reestenose ou oclusão em quatro doentes $(5,3 \%)$.

Conclusão: A endarterectomia carotídea pode ser realizada com segurança no doente em vigília, utilizando-se o shunt seletivamente, com a utilização de fármaco-proteção cerebral. 0 bom resultado obtido e a baixa morbimortalidade trazem um diferencial no tratamento da doença carotídea.

Palavras-chaves: endarterectomia das carótidas, anestesia local, doença cerebrovascular.

\section{Abstract}

O bjective: The purpose of this study is to evaluate the results of carotid endarterectomy performed under locoregional anesthesia with invraoperative cerebral protection and selective shunting.

Method: We reviewed 107 patients who underwent carotid endarterectomy under locoregional anesthesia, at $\mathrm{H}$ ospital das Clínicas da U niversidadeE stadual de Campinas (U N ICAM P), between M arch 1996 and $M$ ay 2004. T his study is composed of 119 endarterectomies (108 patients), being 69 male (63.8\%) and 39 female (36.2\%). M ean age was 63 years, ranging from 43 to 83 years. Surgery was performed to treat symptomatic stenosis in 60 patients $(55.5 \%)$ and asymptomatic stenosis in 48 patients (44.5\%). All patients were assessed by color-flow duplex scanning and arteriography.

Results: Five patients were excluded from the study, since they required conversion to general anesthesia due to intoleranceto carotid clamping. Of the 103 patients submitted to 114 endarterectomies, a shunt was inserted in four patients $(3.5 \%)$, due to the patient's intolerance to carotid clamping during the surgery. A patch was applied in 19 patients (16.6\%). M ortality rate in the immediate postoperative period was $2.6 \%$ (three patients), all due to vascular cerebral accident. O ne patient $(0.87 \%)$ presented a lower ischemic cerebral vascular accident, and one patient $(0.87 \%)$ presented hyperfusion syndrome, which progressed to a hemorrhagic cerebral vascular accident. This patient was submitted to aspiration of the cerebral hematoma, with full recovery of the neurological deficit. In the late postoperative period, there was restenosis or occlusion in four patients $(5.3 \%)$.

Conclusion: Carotid endarterectomy can be safely performed in the awake patient, by selective shunting and cerebral protection. Good outcome and low morbidity and mortality rate bring a differential in the treatment of the carotid disease.

Key words: carotid endarterectomy, local anesthesia, cerebrovascular disease.

1. Doutora. Professora, Disciplina de Moléstias Vasculares, Departamento de Cirurgia, Faculdade de Ciências Médicas, Hospital das Clínicas, Universidade Estadual de Campinas (UNICAMP). Coordenadora, Centro de Referência de Alta Complexidade em Cirurgia Endovascular.

2. Residentes da Disciplina de Moléstias Vasculares, Departamento de Cirurgia, Faculdade de Ciências Médicas, Hospital das Clínicas, UNICAMP, Campinas, SP.

3. Médico contratado, Hospital das Clínicas, UNICAMP. Membro do Centro de Referência de Alta Complexidade em Cirurgia Endovascular

Artigo submetido em 29.04.05, aceito em 17.08.05. 
0 primeiro relato decirurgia corretiva com sucesso para estenose de artéria carótida interna foi descrito em 1954¹. D esdeentão, um número crescentedetrabalhos sobre 0 assunto vem sendo publicado ${ }^{2-5}$, mas somente as grandes séries prospectivas e randomizadas no final da década de 1980 einício dosanos 1990 estabeleceram parâmetros quanto à indicação eao benefício da endarterectomia carotídea6-8. Contudo, al guns aspectos permanecem indefinidose, aindahoje, hácontrovérsiasem relação ao tipo de anestesia, às variações da técnica, à monitorização eproteção cerebral empregada eà necessidade da utilização de shunt.

0 objetivo deste trabalho é descrever e analisar os resultados da endarterectomia de carótida sob an estesia loco-regional, com o uso sel etivo de shunt e a fármacoproteção cerebral.

\section{C asuística e método}

Foram estudados, retrospectivamente, 108 doentes submetidos a 119 endarterectomias carotídeas (11 casos com cirurgia bilateral) com bloqueio loco-regional, no período de maio de 1996 a maio de 2004, no H ospital deC línicas daU niversidadeEstadual deC ampinas (U N ICAM P). A média de idade foi de 63 anos, variando de 43 a 83 anos, sendo 69 pacientes do sexo masculino $(63,8 \%)$ e 39 do sexo feminino $(36,2 \%)$. D esse grupo de doentes, 88 apresentavam hipertensão arterial, 101 eram diabéticos, 85 tabagistas, 36 dislipidêmicos, oito apresentavam insuficiência renal, seis doença pulmonar obstrutiva, 46 doença arterial coronariana isquêmica e 49 doença isquêmica vascular periférica (Figura 1).

Todos os doentes incluídos neste estudo foram aval iados pelo eco-D oppler colorido bilateral, sendo esse exame de imagem utilizado como screening. 0 critério diagnóstico para confirmação e cálculo do grau de estenose foi a angiografia por subtração digital, baseando-se no estudo NASCET ${ }^{6}$, para os sintomáticos, e no $\mathrm{ACAS}^{7}$, para os assintomáticos. Todos os doentes apresentavam estenose de artéria carótida interna igual ou superior a $70 \%$. D ois doentes com contra-indicação para arteriografia, pela utilização de contraste iodado, foram submetidos ao exame de angiorressonância.

Em 31 doentes (28,7\%), a indicação de cirurgia foi antecedente de acidente vascular cerebral isquêmico ( $\mathrm{AVCl}$ ); em 24 doentes (22,2\%), ataque isquêmico transitório (AIT); e em quatro (3,7\%), amaurosefugaz.

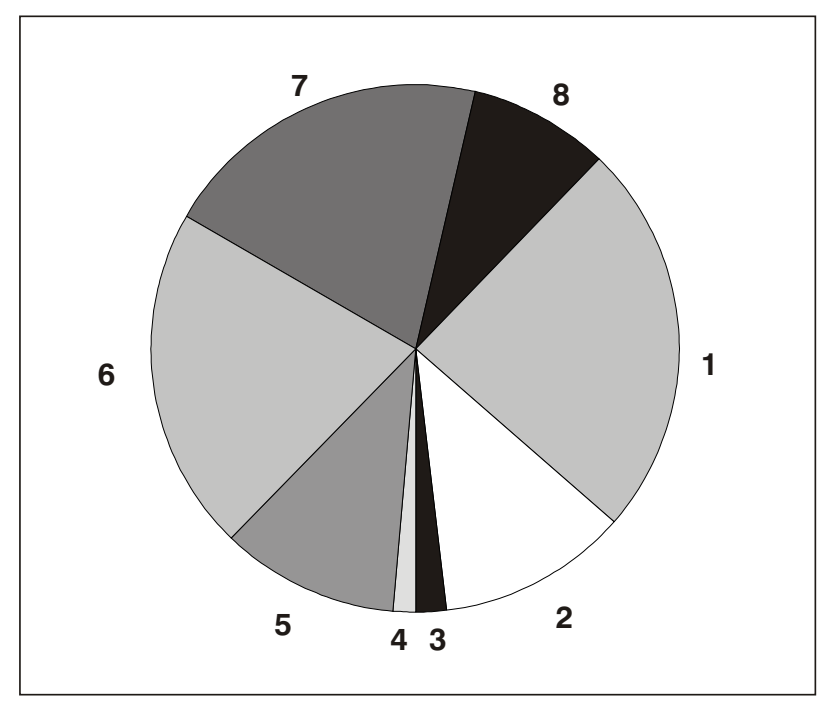
1. Diabetes $93,50 \%$
2. Doença vascular periférica $45,40 \%$
3. Insuficiência renal crônica $7,50 \%$
4. DPOC $5,50 \%$
5. Doença coronariana $42,60 \%$
6. Hipertensão $81,50 \%$
7. Tabagismo $78,70 \%$
8. Dislipidemia $33,30 \%$

Figura 1 - Fatores de risco dos doentes submetidos à endarterectomia de carótida

Em um caso $(0,92 \%)$, a indicação da operação foi reestenose sintomática maior que $80 \%$. 0 s outros 48 pacientes $(44,5 \%)$ eram assintomáticos.

$A$ anestesia realizada foi o bloqueio superficial e profundo do plexo cervical do segundo (C 2) ao quarto (C 4) dermátomoscervicaisesubmandibular, com infiltração de bupivacaína sem vasoconstritor a 0,25\% e bicarbonato de sódio de 2 a $4 \mathrm{ml}$, na emergência dos nervos nos forames cervicais ${ }^{9}$, associado à sedação endovenosa com hipnoanalgésicos.

As medidas de proteção cerebral na anestesia loco-regional seguem o protocolo da nossa equipe de anestesia e iniciam com a orientação do paciente quanto ao método a ser empregado e os sinais de comunicação durante 0 ato cirúrgico (movimentação de membros, fala, al teração de nível de consciência). Por via venosa, administra-se 1 a 2 mg de midazolan e $5 \mathrm{mg} / \mathrm{kg}$ de peso corporal de alfentanil ( $5 \mathrm{mg} / \mathrm{kg}$ peso), sendo que este último é descontínuo 
e de acordo com a demanda. N o início da anestesia, é administrado 1,6 mg de dexametasona.

0 controleda pressão arterial (PA) éfeito através da pressão arterial média (PAM), monitorada de modo invasivo. É realizada a sondagem vesical para controle de diurese, além da utilização de oxigênio contínuo por máscara. A mesa cirúrgica permanece em proclive ou em "canivete". Senecessário, administramosnitroprussiato contínuo na dosagem de 0,05 micrograma $(\mu \mathrm{g})$ por quilo de peso corporal, por minuto, através de bomba de infusão, com o intuito de manter a PA no mesmo nível das medidas queforam obtidas do doente na enfermaria, ou aPAM entre 80 e $120 \mathrm{mmH}$ g. Cinco minutos antes da colocação das pinças arteriais para teste de oclusão carotídea (feito por 3 minutos), são administradas 5000 unidades internacionais (UI) de heparina intra-venosa.

A monitorização cerebral intra-operatória consistiu do exame neurológico e observação de sinais ou sintomas de isquemia cerebral, tais como al terações no nível de consciência, convulsões, alterações da fala ou déficit motor nosmembros. Ao final do ato operatório, sempre revertemos meia dose da heparina administrada com protamina.

As variações técnicas empregadas nas cirurgias foram: 1) endarterectomia convencional e fechamento primário, 2) endarterectomia convencional e fechamento com remendo (patch) e 3) endarterectomia por eversão parcial. 0 shunt foi utilizado deforma seletiva, baseado no statusneurológico duranteo testedeoclusão por 3 minutos ou durante a oclusão carotídea intraoperatória.

A freqüência de avaliação do doente no período pós-operatório foi: no sétimo dia de pós-operatório, 1 mês, 3 meses, 6 meses e anualmente a seguir. $N$ essas consultas, foram pesquisados sinais ou sintomas de isquemia cerebral pós-operatória e, quando presentes, foi indicado exame com eco-D oppler colorido. $\mathrm{N}$ os assintomáticos, o eco-D oppler colorido foi indicado entre 6 meses e 1 ano após a cirurgia. Em caso de estenoses significativas ao eco-D oppler, foi indicada a arteriografia para a confirmação da lesão.

\section{Resultados}

A endarterectomia carotídea foi realizada sob bloqueio loco-regional nos 108 pacientes (119 endarterectomias), tendo sido excluídos cinco doentes $(4,6 \%)$, nos quais o bloqueio foi convertido para anestesia geral e utilização de shunt, devido à intolerância ao teste de oclusão carotídea, em que os doentes apresentaram sinais ou sintomas de isquemia cerebral (doiscasos com convulsões e três com queda do nível de consciência). Foram analisados, portanto, 103 doentes, com 114 endarterectomias realizadas integralmente com anestesia loco-regional.

A técnica operatória real izada foi a endarterectomia carotídea com arteriotomia da carótida interna e sutura primária em 76 casos (66,6\%), endarterectomia carotídea com abertura da artéria carótida interna e fechamento com remendo em 19 delas (16,7\%) e endarterectomia com abertura da artéria carótida comum, bulbo carotídeo e retirada da placa por eversão parcial em 19 doentes (16,7\%).

0 remendo utilizado foi de pericárdio bovino nos 19 casos (16,7\%). A opção por uso de remendo foi feita nos casos em que a artéria carótida interna possuía menos de $5 \mathrm{~mm}$ de diâmetro, no julgamento do cirurgião, quando a incisão da artéria carótida interna se estendia por mais de $3 \mathrm{~cm}$ ou quando a arteriotomia apresentava algum defeito passível de correção com o uso de remendo. A utilização de remendo foi de nove no sexo feminino e 10 no sexo masculino. Foi observado que os remendos aplicados nas carótidas femininas foram mais relacionados a artérias finas (calibre menor que $5 \mathrm{~mm}$ de diâmetro) e nas carótidas masculinas, devido à extensão da arteriotomia (maior que $3 \mathrm{~cm}$ ou defeito no fechamento).

0 shunt de Pruitt-Inahara foi utilizado em quatro doentes $(3,5 \%)$, sendo três $(2,60 \%)$ do sexo feminino e um do sexo masculino $(0,87 \%)$, os quais apresentaram déficit neurológico (queda do nível de consciência) após a realização da arteriotomia.

0 tempo médio depermanência da pinça hemostática na artéria carótida interna foi de 27 minutos (12 a 67 minutos) (T abela 1).

No período pós-operatório imediato, ocorreu AV CI maior e óbito em três $(2,60 \%)$ doentes; outro doente $(0,87 \%)$ apresentou AVCI menor, e um doente $(0,87 \%)$ apresentou síndromedehiperperfusão, que evoluiu com acidente vascular cerebral hemorrágico (AVCH ). E ssedoentefoi submetido à drenagem do hematoma cerebral, com recuperação total do déficit neurológico. Em quatro (3,5\%) operações, ocorreu sangramento da incisão, necessitando de reexploração cirúrgica (T abela 2 ). 
T abela 1 - Indicação de cirurgia e dados operatórios

\begin{tabular}{lr}
\hline D oentes & $108(100 \%)$ \\
Endarterectomias & $119(100 \%)$ \\
Assintomáticos & $48(44,5 \%)$ \\
Sintomáticos & $60(55,5 \%)$ \\
Conversão para anestesia geral & $5(4,6 \%)$ \\
Shunt & $4(3,5 \%)$ \\
Remendo de pericárdio bovino & $19(16,7 \%)$ \\
Fechamento primário & $76(66,6 \%)$ \\
Técnica da eversão & $19(16,7 \%)$ \\
Tempo médio de oclusão por clampe carotídeo & 27 min \\
\hline
\end{tabular}

0 tempo de internação variou entre 2 a 34 dias pós-operatórios, com uma média de 4 dias e mediana de 3 dias.

0 seguimento pós-operatório médio foi de 19 meses (0-98 meses), tendo sido realizado eco-D oppler colorido em $75(72,8 \%)$ doentes. Foi verificada reestenose em dois pacientes $(2,67 \%)$ e oclusão da artéria operada em dois outros casos $(2,67 \%)$. Considerou-se reestenoseasestenosespós-operatóriasmaioresou iguais a $70 \%$, segundo o estudo ultra-sonográfico.

\section{D iscussão}

A endarterectomia carotídea é bastante realizada nos países desenvolvidos, com indicações bem sedimentadas e protocolos estabelecidos ${ }^{2-5,8}$. T em a intenção de reduzir o risco de AVC eóbito edemonstrou ser benéfica na redução desses, tanto em doentes sintomáticos como assintomáticos ${ }^{6,7}$. N o entanto, infelizmente, o AVC ainda é a complicação pós-operatória mais temida desse procedimento. Assim, atenção tem sido dada aos aspectos técnicos da cirurgia, em especial a preservação emonitorização da função cerebral durante o período da aplicação das pinças arteriaisnas carótidas.

A escolha da técnica anestésica pode influenciar o resultado dos procedimentos, e a opção por anestesia loco-regional ${ }^{9}$ ou geral vem sendo motivo de controvérsia desde a introdução dessa técnica ${ }^{1,4}$. Em nosso serviço, optamos pela anestesia loco-regional, por acreditarmos que apresenta vantagens sobre a anestesia geral, permitindo a monitorização neurológica clínica contínua, perioperatória, e diminui a necessidade do uso de shunt. Alguns autores acrescentam como vanta-

T abela 2 - Incidência de acidente vascular cerebral (AVC) eóbito na cirurgia de endarterectomia de carótida

\begin{tabular}{lll}
\hline AVC total pós-operatório & 5 casos & $(4,38 \%)$ \\
AVC isquêmico maior pós-operatório (seguido de óbito) & 3 casos & $(2,6 \%)$ \\
AVC isquêmico menor pós-operatório & 1 caso & $(0,87 \%)$ \\
AVC hemorrágico pós-operatório & 1 caso & $(0,87 \%)$
\end{tabular}


gens, ainda, o menor custo, diminuição do tempo de internação pós-operatório, maior estabilidade hemodinâmica, menor necessidadedeanti-hipertensivosintravenosos e diminuição do tempo cirúrgico ${ }^{10}$. A única grande desvantagem dessa técnica é a intolerância por parte de al guns pacientes.

0 uso deshunt apresentaum potencial deaumentar as complicações da endarterectomia carotídea, podendo lesar a íntima durante sua aplicação, causando trombose precoce ou estenose tardia, além do risco descrito na literatura de embolização aérea e de placas. Portanto, uma vez que acreditamos que o uso de shunt não é totalmente isento de riscos, optamos pelo uso seletivo, diminuindo assim a possibilidade de complicações iatrogênicas do procedimento.

Em relação ao uso de hipnoanalgésicos, acreditamosfacilitar a tolerância do doente ao tempo deoperação, com manutenção da cabeça na mesma posição durante toda a cirurgia, somada à tranqüilidade do doente, quefacilita o controle da PA. Valelembrar que, mesmo com hipnoanalgésicos, o doente atende ao comando verbal do anestesiologista. 0 ptamos, ainda, pela utilização do nitroprussiato, pois proporciona um controlepreciso eimediato daPA. Se, durantea oclusão da carótida pelo clampe, ocorrer um aumento da PA superior a $15 \%$ à pressão inicial do paciente ou PAM superiora $120 \mathrm{mmH}$ g (deveestar entre80 e120 mmH g), a administração do nitroprussiato efetiva o controle prontamente. Lembramos que essa droga é de ação fugaz e de fácil controle.

Preconizamos a utilização de corticosteróide, visando inibir o edema cerebral causado pel o mecanismo da isquemia/reperfusão, que pode comprometer o resultado da operação de restauração vascular ${ }^{11}$. 0 mecanismo de ação do corticóide não está completamente definido, mas se acredita que atuam modulando a ação dosneutrófilosefunção en dotelial, inibindo a liberação de produtos do ácido aracdônico, diminuindo a peroxidação lipídica da membrana e atuando como scavengers de radicais livres ${ }^{12}$.

A reversão da metade da dose da heparina érealizada com protamina, diminuindo, assim, as complicações inerentes ao sangramento local, embora alguns trabal hos naliteratura discutam as complicaçõesno uso da protamina para reversão da heparina ${ }^{13,14}$.

A incidência de reestenose de carótida após endarterectomia tem sido estimada em 1 a 3\%, em pacientes sintomáticos, eem extremos de 10 até $36 \%$, em pacien- tesassintomáticos ${ }^{15}$. N estegrupo, composto por doentes sintomáticos e assintomáticos, a incidência de reestenose ou oclusão foi de $3,57 \%$ e, portanto, semelhante aosresultadosdeoutrostrabal hos ${ }^{16}$. D evemos considerar, ainda, que o resultado do tratamento operatório depende muito da experiência da equipe cirúrgica e anestésica ${ }^{17}$.

Q uando avaliamos os índices de AVC e óbito, assim como as complicações menores desta amostra, encontramos resultados equivalentes aos citados na literatura ${ }^{6,7}$, o que nos permite concluir que a endarterectomia carotídea sob bloqueio loco-regional, com fármaco-proteção cerebral euso sel etivo de shunt éuma técnica cirúrgica com baixos índices de morbimortalidade, trazendo um diferencial no tratamento cirúrgico da doença carotídea, sendo aplicada com segurança em nosso serviço.

\section{R eferências}

1. Eastcott H H, Pickering GW, Rob CG. Reconstruction of internal carotid artery. Lancet. 1954;13:994-6.

2. D eBakey M E, C rawford ES, C ooley D A, M orris GC, G arrett E. Cerebral artery insufficiency: one to 11-year following arterial reconstructive operation. Ann Surg. 1965;921-45.

3. M ovius $\mathrm{HJ}$, Zuber WF, Gaspar MR. Carotid thromboendarterectomy. Arch Surg. 1967;94:585-91.

4. De Bakey ME. Successful carotid endarterectomy for cerebrovascular insufficiency- nineteen-year follow-up. J AM A. 1975;233:1083-5.

5. D eweese JA, Rob CG, Satran R, M arsh DO, Joynt RJ, Summers D, N ichols C. Results of carotid endarterectomies for transient ischemic attacks - five years later. Ann Surg. 1973;178:258-64.

6. N orth American Symptomatic C arotid Endarterectomy T rial Collaborators. Beneficial effect of carotid endarterectomy in symptomatic patientswith high-gradecarotid stenosis. N Eng J M ed. 1991;325:445-53.

7. Executive Committee for the Asymptomatic Carotid Atherosclerosis Study. Endarterectomy for asymptomatic carotid stenosis. JAM A. 1995;273:1421-48.

8. $O$ derich $G S$, Francisconi $A B$, Francisconi $C R$, Pereira $A H$. $M$ omento ideal para a endarterectomia de carótida após um AVC isquêmico. Rev Col Bras Cir. 2001;28:280-4.

9. Gürer $O, Y a p c F, E n c ̧ Y, C ̧$ nar $B, K$ etenci $B, O ̈$ zler $A C$. Local versusgeneral anesthesiafor carotid endarterectomy: report of 329 cases. V asc Endovasc Surg. 2003;37:171-7.

10. Ballotta E, Da Giau GJ. Selective shunting with eversion carotid endarterectomy. V asc Surg. 2003;38;1045-50.

11. Tardini DM, Yoshida W B. Lesões cerebrais decorrentes de isquemia e reperfusão na cirurgia de endarterectomia de carótida. J Vasc Br. 2003;2:119-28. 
12. Korompilas AV, C hen LE, Seaber AV, U rbaniak JR. Actions of glucocorticosteroids on ischemic-reperfused muscle and cutaneous tissue. M icrosurgery. 1997;17:495-502.

13. M auney M C, Buchanan SA, Lawrence W A. Stroke rate is markedly reduced after carotid endarterectomy by avoidance of protamine. J Vasc Surg. 1995;22:264-70.

14. Timi JR, Toregeani JF, M iyamotto M, Ribeiro IG. Complicaçõesperioperatórias em endarterectomia decarótida relacionadas a protamina. J V asc Br. 2003;2:291-5.

15. M oreira RR. Endarterectomia da carótida. Cir V asc Angiol. 1998;14:154-61.

16. G lover JL, Bendick PJ, D illey RS, et al. Restenosis following carotid endarterectomy. Ann Surg. 1985;120:678-84.
17. Feasby TE, Quan H, Ghali WA. Hospital and surgeon determinants of endarterectomy outcomes. Arch $\mathrm{N}$ eurol. 2002;59:1877-81.

Correspondência:

Ana T erezinha Guillaumon

Rua H ermantino C oelho, 901/11

CEP 13087-500 - Campinas, SP

Fax: (19) 3788.7442

E-mail: terag@superig.com.br 